\title{
Wiener Indices of Balanced Binary Trees
}

\author{
Sergey Bereg and Hao Wang \\ Dept. of Computer Science, University of Texas at Dallas, Richardson, TX 75083 \\ \{besp, haowz\}@utdallas.edu
}

\begin{abstract}
We study a new family of trees for computation of the Wiener indices. We introduce general tree transformations and derive formulas for computing the Wiener indices when a tree is modified. We present several algorithms to explore the Wiener indices of our family of trees. The experiments support new conjectures about the Wiener indices.
\end{abstract}

\section{Introduction}

Molecules and molecular compounds are often modeled by molecular graphs. One of the most widely known topological descriptor 6, 10, is the Wiener index named after chemist Harold Wiener [15. The Wiener index of a graph $G(V, E)$ is defined as $W(G)=\sum_{u, v \in V} d(u, v)$, where $d(u, v)$ is the distance between vertices $u$ and $v$ (minimum number of edges between $u$ and $v$ ).

A majority of the chemical applications of the Wiener index deal with chemical compounds that have acyclic organic molecules. The molecular graphs of these compounds are trees [7, see an example of a chemical compound in Fig. 1] Therefore most of the prior work on the Wiener indices deals with trees, relating the structure of various trees to their Wiener indices (asymptotic bounds on the Wiener indices of certain families of trees, expected Wiener indices of random trees etc.). For these reasons, we concentrate on the Wiener indices of trees as well (see Dobrynin et al. [3] for a recent survey).

For trees with bounded degrees of vertices, Jelen and Triesch [11 found a family of trees such that $W(T)$ is minimized. Fischermann et al. 4 ] solved the same problem independently. They characterized the trees that minimize and maximize the Wiener index among all trees of a given size and the maximum vertex degree.

Several papers address the question: What positive integer numbers can be Wiener indices of graphs of a certain type? The question is answered for general graphs and bipartite graphs [3. The question is still open for trees.

Conjecture 1. [Wiener Index Conjecture [5, 9, 12] Except for some finite set, every positive integer is the Wiener index of a tree.

Lepović and Gutman 12 found the Wiener indices up to 1206 by enumerating all non-isomorphic trees of at most 20 vertices. They conjectured that 159 is the largest non-Wiener index of a tree. Goldman et al. [5] verified the conjecture for 


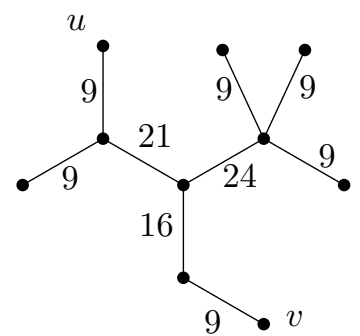

Fig. 1. Carbon skeleton of 3-Ethyl-2,2,4-trimethylpentane. Its Wiener index is 115 which is $W(G)=\sum_{u, v \in V} d(u, v)$ (for example, $d(u, v)=4$ ), and $W(G)=$ $\sum_{e \in T} n_{1}(e) n_{2}(e)$ where $n_{1}(e)$ and $n_{2}(e)$ are the sizes of two trees left after the removal of $e$

the Wiener indices up to $10^{4}$. Recently Ban et al. 1 found a class of trees whose Wiener indices cover all numbers up to $10^{8}$. Although their algorithm is very fast, the trees may have vertices of large degrees.

The molecular graphs have bounded degrees. In fact, a molecular graph, whose vertices represent atoms and edges represent chemical bonds, has maximum vertex degree 4 [14. In this paper we study a new class of trees that possess useful properties such as (i) the vertex degrees are bounded, and (ii) the trees are balanced, and (iii) they admit efficient algorithms for computing Wiener indices.

We define a $k$-tree, $k=0,1,2, \ldots$ as a rooted binary tree such that (i) every node of depth less than $h-k$ has exactly two children where $h$ is the height of the tree, and (ii) a node of depth at least $h-k$ at most two $(0,1$ or 2$)$ children. Let $\mathcal{F}_{k}$ denote the family of all $k$-trees. $\mathcal{F}_{k}, k=0,1,2 \ldots$ is a growing family of trees since $\mathcal{F}_{0} \subset \mathcal{F}_{1} \subset \mathcal{F}_{2} \subset \ldots$

Let $\mathcal{F}_{k}(n)$ denote the set of $k$-trees of size $n$. Let $W\left(\mathcal{F}_{k}\right)$ and $W\left(\mathcal{F}_{k}(n)\right)$ denote the set of the Wiener indices of the trees in $\mathcal{F}_{k}$ and $\mathcal{F}_{k}(n)$, respectively. The family $\mathcal{F}_{0}$ contains the complete binary trees only and $W\left(\mathcal{F}_{0}\right)$ is a sequence of numbers (defined as a function of the tree height) that grow exponentially. Therefore the Wiener indices of trees of $\mathcal{F}_{0}$ cannot justify Conjecture 1. We present efficient algorithms for computing the Wiener indices of $\mathcal{F}_{k}(n)$ for $k=$ 1,2 and general $k$. We implemented the algorithms for $k=1$ and $k=2$. We found all Wiener indices of $W\left(\mathcal{F}_{1}\right)$ up 7001724 and $W\left(\mathcal{F}_{2}\right)$ up to 30224. Our experiments allow us to suggest the following.

Conjecture 2. Except for some finite set, every positive integer is the Wiener index of a binary tree.

\section{$2 \quad$ Preliminaries}

Canfield et al. 2] applied a recursive approach for calculating the Wiener index of a tree. For a rooted tree $T$, we denote by $l(T)$ the sum of the distances from the root $v_{\text {root }}$ of $T$ to all its vertices, $l(T)=\sum_{v \in T} d\left(v_{\text {root }}, v\right)$. 
Theorem 3 (Canfield et al. [2]). Let $T$ be a tree of size $n$ with the root $v_{\text {root }}$ and let $v_{i}, 1 \leq i \leq k$ be the vertices adjacent to $v_{\text {root }}$. Let $T_{i}, 1 \leq i \leq k$ be the subtree of $T$ rooted at $v_{i}$. Let $n_{i}$ be the size of $T_{i}, 1 \leq i \leq k$. Then

$$
\begin{aligned}
W(T) & =n(n-1)+\sum_{i=1}^{k}\left[W\left(T_{i}\right)+\left(n-n_{i}\right) l\left(T_{i}\right)-n_{i}^{2}\right] \\
l(T) & =n-1+\sum_{i=1}^{k} l\left(T_{i}\right) .
\end{aligned}
$$

Wiener [15] discovered the following formula $W(G)=\sum_{e \in T} n_{1}(e) n_{2}(e)$ where $n_{1}(e)$ and $n_{2}(e)$ are the sizes of two trees left after the removal of $e$, see Fig. 1.

\section{Bounds for $k$-Trees}

We derive bounds for $k$-trees that are useful in further analysis. It is interesting that, for a fixed $k$, the difference between the Wiener indices of $k$-trees of size $n$ is $O\left(n^{2}\right)$ though the Wiener indices themself are bounded by $O\left(n^{2} \log n\right)$ (these bounds are tight!).

Theorem 4. Let $T$ and $T^{\prime}$ be two k-trees of size $n$. Then

$l(T) \leq n(k-1+\log (n+1))$ and $W(T) \leq n(n-1)(k-1+\log (n+1))$

$\left|l(T)-l\left(T^{\prime}\right)\right| \leq(2 k-1) n$ and $\left|W(T)-W\left(T^{\prime}\right)\right| \leq 2\left(\left(2^{k}-1\right) n^{2}+4^{k}(n+1)^{2}\right)$.

\section{Tree Operations}

In this Section we introduce two operations on general rooted trees and derive formulas for the Wiener index. Let $T$ be a rooted tree. For a node $v$ of $T$, we denote the subtree rooted at $v$ by $T(v)$ and its size by $n(v)$. We consider two operations on the tree $T$

- swapping subtrees where two subtrees of $T$ rooted at nodes $v$ and $u$ are switched, see Fig. 2 (b), and

- joining subtrees where a subtree rooted at $v$ moves to a vertex $u$, see Fig. $Q$ (c). Note that this operation applied to a binary tree can produce a non-binary tree. The resulting tree is binary iff $v$ and $u$ have at most two children together.

We derive formulas for calculating the Wiener index when a tree is modified.

Theorem 5 (Tree operations). Let $u$ and $v$ be two nodes of $T$ with the same depth. Let $w$ be the lowest common ancestor of $u$ and $v$. Let $u_{0}=w, u_{1}, u_{2}, \ldots, u_{k}$ $=u$ be the path between $w$ and $u$ and let $v_{0}=w, v_{1}, v_{2}, \ldots, v_{k}=v$ be the path between $w$ and $v$. Let $\Delta=n(v)-n(u)$ be the difference of the sizes of two subtrees $T(v)$ and $T(u)$. 


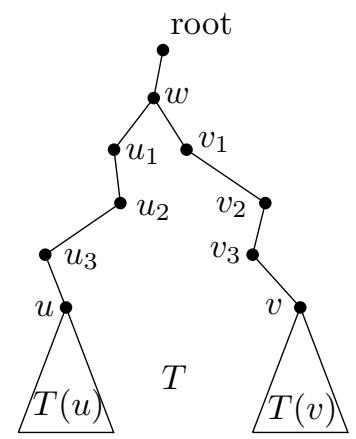

(a)

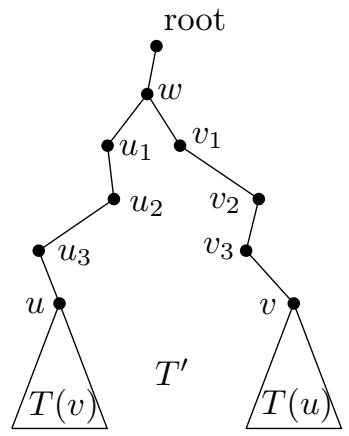

(b)

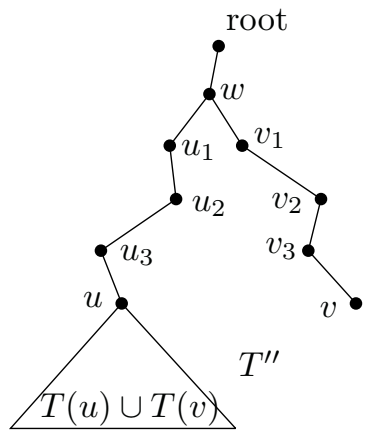

(c)

Fig. 2. Tree operations. (a) Original tree $T$. (b) Swapping the trees $T(u)$ and $T(v)$. (c) Joining the trees $T(u)$ and $T(v)$

I. Let $T^{\prime}$ be the tree obtained by swapping the subtrees $T(v)$ and $T(u)$ in $T$, see Fig. 2 (b). Then

$$
W\left(T^{\prime}\right)=W(T)-2 k \Delta^{2}+2 \Delta \sum_{i=2}^{k}\left(n\left(v_{i}\right)-n\left(u_{i}\right)\right) .
$$

II. Let $T^{\prime \prime}$ be the tree obtained by joining the subtrees $T(v)$ and $T(u)$ in $T$, see Fig. Q (c). Then

$$
W\left(T^{\prime \prime}\right)=W(T)-2 k \cdot n^{2}(v)+2 n(v) \sum_{i=1}^{k}\left(n\left(v_{i}\right)-n\left(u_{i}\right)\right) .
$$

\section{1-Trees}

We show that the Wiener indices of 1-trees can be computed efficiently. For a given number $n$, there are exponentially many 1-trees of size $n$ (there are actually exponentially many non-isomorphic rooted 1-trees). By Theorem 4 the Wiener indices are bounded by a polynomial function. We observe the following property of 1-trees that enables a polynomial time algorithm for computing $W\left(\mathcal{F}_{1}(n)\right)$.

Lemma 1. Let $T$ be a 1-tree of size $n$. The height of $T$ is $h=\lfloor\log n\rfloor$.

(i) The value $l(T)$ is a function of $n$, denoted $l(n)$, and can be calculated as follows

$$
l(n)=h(n+1)-2^{h+1}+2 .
$$

(ii) Let $T_{1}$ and $T_{2}$ be two trees obtained by removing the root from $T$. Let $n_{i}=\left|T_{i}\right|, i=1,2$. Then $n_{1}+n_{2}=n-1$ and

$$
W(T)=W\left(T_{1}\right)+n_{1}+l\left(n_{1}\right)+W\left(T_{2}\right)+n_{2}+l\left(n_{2}\right)+l\left(n_{1}\right) n_{2}+l\left(n_{2}\right) n_{1}+2 n_{1} n_{2} .
$$


Lemma 1 provides an efficient way to compute the Wiener indices. We represent a group of 1-trees of size $n$ with the same Wiener index $w$ by just the pair $(w, n)$. Note that there can be exponentially many 1 -trees for the same pair $(w, n)$. Lemma 1 allows us to create a new group of trees $(w, n)$ from two groups $\left(w_{i}, n_{i}\right), i=1,2$. Every tree in the group $(w, n)$ is a combination of two trees from groups $\left(w_{1}, n_{1}\right)$ and $\left(w_{2}, n_{2}\right)$ respectively.

We compute the set $W\left(\mathcal{F}_{1}(n)\right)$ using dynamic programming. We assume that the sets $W\left(\mathcal{F}_{1}(i)\right), i<n$ are computed. We store $L$, a sorted list of numbers that are the Wiener indices found so far (initially $L$ is empty). For all $n_{1}=1, \ldots,\lceil(n-$ $1) / 2\rceil$ and all two numbers $w_{1} \in W\left(\mathcal{F}_{1}\left(n_{1}\right)\right)$ and $w_{2} \in W\left(\mathcal{F}_{1}\left(n_{2}\right)\right), n_{2}=n-n_{1}-$ 1 , the algorithm does the following. First, it checks whether $T$, the combination of two 1-trees $T_{1}$ and $T_{2}$ corresponding to the pairs $\left(w_{1}, n_{1}\right)$ and $\left(w_{2}, n_{2}\right)$, is a valid 1-tree. We compute the heights $h_{i}=\left\lfloor\log n_{i}\right\rfloor, i=1,2$. If $h_{1}=h_{2}$ then $T$ is a valid 1-tree. If $\left|h_{1}-h_{2}\right| \geq 2$ then $T$ is not a valid 1-tree. Suppose that $\left|h_{1}-h_{2}\right|=1$. We can assume for simplicity that $h_{1}<h_{2}$. Then $T$ is a valid 1-tree if and only if $T_{1}$ is a complete binary tree.

If $T_{1}$ and $T_{2}$ can be combined, we compute $w$ by formula (6) and check if $w \in L$ in $O(\log n)$ time. If $w$ is a new Wiener index, then it is inserted in $L$. The sizes of $W\left(\mathcal{F}_{1}\left(n_{1}\right)\right)$ and $W\left(\mathcal{F}_{1}\left(n_{2}\right)\right)$ are bounded by $O\left(n^{2}\right)$ by Theorem 4 . Thus, the total time for computing $W\left(\mathcal{F}_{1}(n)\right)$ is $O\left(n^{5} \log n\right)$.

Theorem 6. The Wiener indices of 1-trees of size at most $n$ can be found in $O\left(n^{6} \log n\right)$ time.

We implemented the above algorithm and run it up to $n=1000$. The running time is 36 hours on Intel processor $2.4 \mathrm{GHZ}, 512 \mathrm{MB}$ memory using Microsoft $\mathrm{C}++6.0$. As we show later the Wiener indices of $W\left(\mathcal{F}_{1}(n)\right), n>1000$ are greater than 7001724. It turns out that there are still many integer numbers close to 7001724 that are not in $W\left(\mathcal{F}_{1}\right)$, for example, the numbers from the interval [6988865,7016907] are not in $W\left(\mathcal{F}_{1}\right)$.

In what follows we explore the Wiener indices $W\left(\mathcal{F}_{1}(n)\right)$ for larger $n$ (more than 1000) based on the minimum and maximum Wiener indices in $W\left(\mathcal{F}_{1}(n)\right)$. We find shapes of 1-trees that have the smallest/largest Wiener indices in $W\left(\mathcal{F}_{1}(n)\right)$. We discuss it in the next section.

\section{$6 \quad$ Interval Method}

Since it is not feasible to compute the Wiener indices $W\left(\mathcal{F}_{1}(n)\right)$ for large $n$, we want to compute intervals $\left[W_{\min }(n), W_{\max }(n)\right]$ for large $n$ where $W_{\min }(n)$ and $W_{\max }(n)$ are the minimum and maximum Wiener indices of $W\left(\mathcal{F}_{1}(n)\right)$, respectively. First, we derive formulas for $W_{\min }(n)$ and $W_{\max }(n)$. We need some notations. Let $v$ be a vertex of a 1-tree $T$. Let $v_{l}$ and $v_{r}$ denote its left child (if any) and its right child (if any), respectively. If $v$ does not have a left/right child we use a dummy vertex $v_{n i l}$ instead. We assume that $n\left(v_{n i l}\right)=0$. 


\subsection{Minimum Wiener Indices}

Let $m$ be a positive integer and let $h(m)=\lfloor\log (m+1)\rfloor$. A pair of positive integers $\left(m_{1}, m_{2}\right)$ is a partition of $m$ if $m_{1}+m_{2}=m$. We call a partition $\left(m_{1}, m_{2}\right)$ of $m$ complete if one of the numbers $m_{i}(i=1,2)$ is $2^{j}-1$ where $j \in\{h(m)-1, h(m)\}$. It can be verified that the number $m_{3-i}$ lies in the interval $\left[2^{h(m)-1}-1,2^{h(m)}-1\right]$. Also, $m$ has a unique complete partition (note that both $m_{1}=2^{h(m)-1}-1$ and $m_{2}=2^{h(m)}-1$ are possible for some $m$ ). Let $\mu(m)$ be the smallest $m_{i}, i=1,2$ of the complete partition of $m$.

Let $v$ be a vertex of a 1-tree $T$. We call $v$ an complete vertex if $\left(n\left(v_{l}\right), n\left(v_{r}\right)\right)$ is the complete partition of $n(v)-1$. Note that, if $v$ is complete, then at least one of the subtrees $T\left(v_{l}\right)$ or $T\left(v_{r}\right)$ is complete. Let

$$
F\left(n_{1}, n_{2}\right)=l\left(n_{1}\right)+l\left(n_{2}\right)+l\left(n_{1}\right) n_{2}+l\left(n_{2}\right) n_{1}+2 n_{1} n_{2}+n_{1}+n_{2} .
$$

Theorem 7. A 1-tree $T$ of $n$ vertices has the minimum Wiener index $W_{\min }(n)$ if and only if every vertex of $T$ is complete. The sequence $W_{\min }(n)$ satisfies the following recurrence:

$$
\begin{gathered}
W_{\min }(1)=0, W_{\min }(2)=1, \\
W_{\min }(n)=W_{\min }\left(n_{1}\right)+W_{\min }\left(n_{2}\right)+F\left(n_{1}, n_{2}\right) \quad \text { if } n \geq 3, \\
\text { where } n_{1}=\mu(n-1), n_{2}=n-n_{1}-1 .
\end{gathered}
$$

\subsection{Maximum Wiener Indices}

We call an internal node $v$ of a binary tree balanced if $\left|n\left(v_{l}\right)-n\left(v_{r}\right)\right| \leq 1$. The following theorem characterizes 1-tree maximizing the Wiener index.

Theorem 8. A 1-tree $T$ of $n$ vertices has the maximum Wiener index $W_{\max }(n)$ if and only if every vertex of $T$ is balanced. The sequence $W_{\max }(n)$ satisfies the following recurrence:

$$
\begin{aligned}
& W_{\max }(1)=0, W_{\max }(2)=1, \\
& W_{\max }(n)=W_{\max }\left(n_{1}\right)+W_{\max }\left(n_{2}\right)+F\left(n_{1}, n_{2}\right), \\
& \text { where } n_{1}=\left\lceil\frac{n-1}{2}\right\rceil, n_{2}=n-n_{1}-1 \text { if } n \geq 3 .
\end{aligned}
$$

Theorem 9. The functions $l(n), W_{\min }(n)$ and $W_{\max }(n)$ are monotonically increasing.

\subsection{Algorithm and Experiments}

We implemented a simple algorithm GAP for finding maximal intervals not covered by the intervals $I_{n}, n \geq 1$. The algorithm is based on the monotonicity of $W_{\min }(n), W_{\max }(n)$ and has linear running time.

We run it for $n \leq 14075$ (the value of $W_{\max }(n)$ exceeds the maximum integer value stored in 32 bits, unsigned long integer type). The largest number not 
covered by intervals is 705344327. Using another property of the Wiener indices we can find even larger numbers not covered by intervals. The Wiener index of a tree with even/odd number of vertices is odd/even, respectively (see for example [5] p. 228). Therefore the intervals $I_{n}$ for even/odd $n$ can cover only odd/even integer numbers. We run our algorithm for even $n$ and odd $n$ separately. The largest gap we found is the interval [722813799,722814221] of odd numbers which sandwiched between $I_{8472}$ and $I_{8474}$. We believe that (i) the intervals $I_{n}$ for all even $n \geq 8474$ cover all odd integers larger than 722814221, and (ii) the intervals $I_{n}$ for all odd $n \geq 8474$ cover all even integers larger than 722814221 .

It is an interesting open question whether there is only a finite number of integers not covered by $W\left(\mathcal{F}_{1}\right)$. We were unable to answer it. Since we found large integer numbers not in $W\left(\mathcal{F}_{1}\right)$, we decided to explore 2-trees that are less computationally attractive.

\section{$7 \quad$ Algorithm for $k$-Trees}

We assume that $k$ is a constant. Let $W\left(\mathcal{F}_{k}(n)\right)$ be the set of the Wiener indices of $k$-trees of size $n$. We present an algorithm for computing $W\left(\mathcal{F}_{k}(n)\right)$, $k \geq 2$. Here we do not have the property that $l(T)$ is a function of $n$. In order to be able to generate many $k$-trees (for large $n$ ) we want to store minimum amount of information that allows us to compute Wiener indices recursively. Let $h(T)$ denote the height of a tree $T$. For a $k$-tree $T$, we define $h c(T)$ as the largest number $h^{\prime}$ such that the vertices of $T$ of height at most $h^{\prime}$ form a complete binary tree. We group $k$-trees with the same $W(T), l(T), h(T)$ and $h c(T)$. We store a list $L_{k}(n)$ of groups $(w, l, h, h c)$ sorted in the lexicographical order.

We compute $L_{k}(n)$ using dynamic programming. We assume that $L_{k}(i), i<$ $n$ are computed. We store elements of $L_{k}(n)$ in lexicographical order. For all $n_{1}=1, \ldots,\lceil(n-1) / 2\rceil$ and all two tuples $t_{1}=\left(w_{1}, l_{1}, h_{1}, h c_{1}\right) \in L_{k}\left(n_{1}\right)$ and $t_{2}=\left(w_{2}, l_{2}, h_{2}, h c_{2}\right) \in L_{k}\left(n_{2}\right)$, the algorithm does the following. First, it checks whether $T$, the combination of two 1-trees $T_{1}$ and $T_{2}$ corresponding to $t_{1}$ and $t_{2}$, is a valid $k$-tree. We compute the heights $h(T)=1+\max \left(h_{1}, h_{2}\right)$ and $h c(T)=$ $1+\min \left(h c_{1}, h c_{2}\right)$. The tree $T$ is a valid $k$-tree if and only if $h(T) \leq h c(T)+k$.

If $T_{1}$ and $T_{2}$ can be combined, we compute $w$ by Equation (1). We check if $t=(w, l, h, h c) \in L_{k}(n)$ in $O(\log n)$ time. If $t$ is a new element it is inserted in $L_{k}(n)$. The number of the Wiener indices of $k$-trees of size $n$ is bounded by $O\left(n^{2}\right)$ by Theorem 4. The heights $h$ and $h c$ of $k$-trees of size $n$ are bounded by $O(k+\log n)=O(\log n)$. The number of $l$-values of trees of $\mathcal{F}_{k}(n)$ is bounded by $O(n)$ by Theorem 4 . Thus, the sizes of $L_{k}\left(n_{1}\right)$ and $L_{k}\left(n_{2}\right)$ are bounded by $O\left(n^{3}\right)$. Therefore the total time for computing $L_{k}(n)$ is $O\left(n^{6} \log n\right)$.

Theorem 10. The Wiener indices of $k$-trees of size at most $n$ can be found in $O\left(n^{7} \log n\right)$ time. 


\section{2-Trees}

For 2-trees we can store just three numbers to represent a group: $w$, the Wiener index, and $b$, the number of vertices at the bottom level (maximum depth vertices). If we remove $b$ vertices at the bottom level from a tree $T$, we obtain a 1-tree $T^{\prime}$. By lemma 1, $l\left(T^{\prime}\right)$ can be computed using the number of vertices of $T^{\prime}$. One can derive formulas for computing $l(T), h(T), h c(T)$. In this way we can speed up the algorithm for generating $L_{2}(n)$.

We implemented the above algorithm and computed the Wiener indices of 2-trees of size up to 90. The integer numbers between 8864 and 30224 are all covered by $W\left(\mathcal{F}_{2}\right)$. The largest integer in $[1,30224] \notin W\left(\mathcal{F}_{2}\right)$ is 8863 . We believe that all numbers larger than 8863 are covered by $W\left(\mathcal{F}_{2}\right)$.

Conjecture 11. The set of Wiener indices $W\left(\mathcal{F}_{2}\right)$ contains all integer numbers greater than 8863.

We compute the density of the Wiener indices $\delta: \mathbb{R} \rightarrow[0,1]$ defined as follows. For a number $x \in \mathbb{R}$, the value $\delta(x)$ is $N / x$ where $N$ is the number of the Wiener indices of $W\left(\mathcal{F}_{2}\right)$ less than $x$. To compute the density function we use a boolean array $B[]$. The boolean value $B[i]$ indicates existence of 2 -tree $T$ such that $W(T)=i$. The density is plotted in Fig. 3. The plot can be viewed as a supporting argument for Conjecture 11]

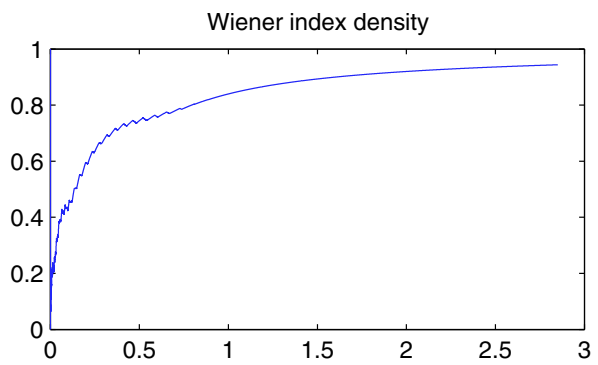

Fig. 3. Density of $W\left(\mathcal{F}_{2}\right)$ for $n \leq 90$. The $x$-values are given in scale 10000 . The $y$-axis is the density

\section{References}

1. A. Ban, S. Bereg, and N. Mustafa. On a conjecture of Wiener indices in computational chemistry. Algorithmica, 40(2):99-118, 2004.

2. E. R. Canfield, R. W. Robinson, and D. H. Rouvray. Determination of the Wiener molecular branching index for the general tree. J. Computational Chemistry, 6:598 609, 1985.

3. A. A. Dobrynin, R. Entringer, and I. Gutman. Wiener index of trees: Theory and applications. Acta Applicandae Mathematicae, 66:211-249, 2001. 
4. M. Fischermann, A. Hoffmann, L. S. Dieter Rautenbach, and L. Volkmann. Wiener index versus maximum degree in trees. Discrete Applied Mathematics, 122(13):127-137, 2002.

5. D. Goldman, S. Istrail, G. L. A., and Piccolboni. Algorithmic strategies in combinatorial chemistry. In Proc. 11th ACM-SIAM Sympos. Discrete Algorithms, pp. 275-284, 2000.

6. R. Gozalbes, J. Doucet, and F. Derouin. Application of topological descriptors in QSAR and drug design: history and new trends. Current Drug Targets: Infectious Disorders, 2:93-102, 2002.

7. I. Gutman and O. E. Polansky. Mathematical concepts in organic chemistry. Springer-Verlag, Berlin, 1986.

8. I. Gutman and J. J. Potgieter. Wiener index and intermolecular forces. J. Serb. Checm. Soc., 62:185-192, 1997.

9. I. Gutman, Y.-N. Yeh, and J. C. Chen. On the sum of all distances in graphs. Tamkang J. Math., 25, 1994.

10. O. Ivanciuc. QSAR comparative study of Wiener descriptor for weighted molecular graphs. J. Chem. Inf. Compuc. Sci., 40:1412-1422, 2000.

11. F. Jelen and E. Triesch. Superdominance order and distance of trees with bounded maximum degree. Discrete Applied Mathematics, 125(2-3):225-233, 2003.

12. M. Lepović and I. Gutman. A collective property of trees and chemical trees. J. Chem. Inf. Comput. Sci., 38:823-826, 1998.

13. D. H. Rouvray. Should we have designs on topological indices?, pp. 159-177. Elsevier, Amsterdam, 1983.

14. N. Trinajstic. Chemical Graph Theory. CRC Press, 1992.

15. H. Wiener. Structural determination of paraffin boiling points. J. Amer. Chem. Soc., 69:17-20, 1947. 